\title{
Routing hazardous material by compulsory check points in case of variable demand
}

\author{
Paola Cappanera ${ }^{1}$ \\ Dipartimento di Ingegneria dell'Informazione \\ Università degli Studi di Firenze \\ Firenze, Italy \\ Maddalena Nonato $^{2}$ \\ Dipartimento di Ingegneria \\ Università degli Studi di Ferrara \\ Ferrara, Italy \\ Filippo Visintin ${ }^{3}$ \\ Dipartimento di Ingegneria Industriale \\ Università degli Studi di Firenze \\ Firenze, Italy
}

\begin{abstract}
The Gateway Location Problem is the core of a new system for Hazardous Material routing, whose risk mitigation potentials have been assessed in case of static demand. We investigate the impact of flexibility in gateway location and allocation decisions in case of variable demand, and provide a tool to support decision makers in establishing a trade off between infrastructure costs and risk mitigation targets.

Keywords: hazardous material transportation, gateway location, variable demand.
\end{abstract}




\section{Introduction}

Hazardous materials, HazMat in the following, are pervasive in our everyday life. They range from petrol products employed in the oil industry to hospital radioactive waste. Since contamination causes severe harm to human health and to the environment, stocking and handling procedures are subject to strict safety guidelines. However, the most dangerous step in HazMat life cycle is transportation. An accident on route is a Low-Probability-High-Consequence event and much research effort has been devoted to risk mitigation policies involving risk assessment and routing. Here we focus on the latter. A high percentage of shipments are transported by truck on short mileage trips, possibly crossing densely populated areas, such as a tank leaving a refinery heading for a petrol station according to a full drop delivery pattern. Since the majority of damages and injures are due to accidents which occur in transit, itinerary related decisions offer a relevant opportunity for risk reduction and control. General laws exist that recommend not to operate the vehicle over routes which go through heavily populated areas, tunnels, narrow streets, or alleys. However, several exceptions are permitted and when no restriction is enforced the driver can prepare the route plan himself and take decisions guided by operating convenience. All this calls for risk-mitigation, safety-aware, route planning procedures [1]. Several methodologies have been proposed to compute the safest itinerary for a single trip, solving so called local routing problems usually faced by individual carriers. From a system perspective, the governmental authority addresses a global routing problem seeking a set of routes, one for each trip, which conjugate risk mitigation and fairness by incorporating equity into the spatial distribution of the individual HazMat routes. When specific itineraries can not be enforced, a rule-based system establishes mandatory directions carriers must follow, leaving a certain degree of freedom to drivers. Challenging network optimization problems arise in this framework ([3]), characterized by a hierarchical relation between different stakeholders with conflicting objectives: authority is driven by risk minimization while drivers pursue cost minimization. A system based on routing by mandatory check points was recently proposed and the related bilevel combinatorial optimization problem, namely the gateway location problem (GLP), was analyzed. In this system, the required infrastructure is light and cheap, as it consists of a set of devices, so called gateways, able to record vehicle's

$\overline{1 \text { Email: }}$ paola.cappanera@unifi.it

2 Email: maddalena.nonato@unife.it

3 Email: filippo.visintin@unifi.it 
identity. GLP consists of properly locating a given number of gateways on the network and to assign one gateway to each vehicle, to be crossed on its route from origin to destination. Previous studies $([4,5])$ suggest that a limited number of gateways allows to achieve high levels of risk mitigation in case of static demand, and cost deterioration is modest [6]. This study is a follow-up of the above research, overcoming the static demand assumption. The more realistic vision taken here considers variable demand over a given number of time periods. We analyze how the system reacts to demand variations with respect to different degrees of flexibility of the infrastructure, i.e. updating gateway location and allocation decisions, in case future demand is known one period at a time. Since changes do not come for free, we believe that this tool can support decision makers in establishing the best compromise between infrastructure costs and risk mitigation targets.

The rest of the paper is organized as follows: In Section 2 we first recall the basic features of the gateway based system in the static demand case. Then, we consider a dynamic framework where the planning horizon is divided into several periods and demand distribution is known. In Section 3 the impact of infrastructure flexibility is experimentally evaluated and discussed, and work in progress is sketched.

\section{Routing vehicles by compulsory check points}

GLP aims at diverting vehicles away from their risky shortest path from origin to destination by assigning to each vehicle a compulsory gateway to be crossed along the itinerary. Apart from this requirement there is no other compliance so that the vehicle will follow the shortest route which goes through the assigned gateway. Formally, this relation is captured by a bilevel optimization MILP model, whose static demand formulation is recalled hereafter. Consider a set of vehicles $V=\{1 \ldots, n\}$, each supplying hazmat from an origin $o_{v}$ to a destination $d_{v}$. The road network is modeled as an oriented graph $G=(N, A)$, with $o_{v}, d_{v} \in N \forall v \in V$. Cost and risk coefficients $c_{i j}>0, r_{i j} \geq 0$ are known $\forall(i, j) \in A$. Consider a generic period $t$ and let $\varphi_{v}^{t}$ be the quantity shipped by $v$ from $o_{v}$ to $d_{v}$ during $t$. The location of $k$ gateways $(k<<n)$ must be selected in $N$ and each vehicle must be assigned to one gateway so that the total risk of the new routes is minimized. Let $g t w(t, v)$ denote the gateway assigned to vehicle $v$ at $t$. Once a gateway has been located at node $h$ and gateway $h$ has been assigned to $v(g t w(t, v)=h)$, then $v$ will travel along the shortest gateway path with respect to $h$. This is made of two paths, the upstream (downstream) gateway path $\bar{p}_{v}^{h}\left(\underline{p}_{v}^{h}\right)$, i.e., the shortest path from $o_{v}$ 
to $h$ (from $h$ to $d_{v}$ ). GLP at time $t$ can be formalized as the problem of selecting a subset $N^{t} \subseteq N$ of $k$ nodes and assigning to each vehicle $v$ one gateway $h \in N^{t}$ so that the sum over each vehicle of the risk of the two paths $\underline{p}_{v}^{g t w(t, v)}$ and $\bar{p}_{v}^{g t w(t, v)}$ is minimized. A concise formulation follows, for details see [4].

$$
\begin{array}{cr}
P^{G T W}(t): \min \sum_{v=1 \ldots n} \varphi_{v}^{t} \sum_{(i, j) \in A} r_{i j}\left(\bar{x}_{i j}^{t, v}+\underline{x}_{i j}^{t, v}\right) & \text { subject to: } \\
\sum_{h \in N} z_{h}^{t, v}=1 & \forall v \in V \\
y_{h}^{t} \geq z_{h}^{t, v} & \forall h \in N, \forall v \in V \\
\sum_{h \in N} y_{h}^{t}=k & \\
z_{h}^{t, v} \in\{0,1\} & \forall h \in N, \forall v \in V \\
y_{h}^{t} \in\{0,1\} & \forall h \in N \\
\bar{x}_{i j}^{v} \in \operatorname{argmin} \overline{S P}_{v}^{t} & \forall v \in V \\
\underline{x}_{i j}^{v} \in \operatorname{argmin} \underline{S P_{v}^{t}} & \forall v \in V
\end{array}
$$

where at period $t: y_{h}^{t}$ denotes an open gateway at node $h ; z_{h}^{t, v}$ assigns gateway $h$ to $v\left(z_{h}^{t, v}=1\right.$ iff $\left.g t w(t, v)=h\right)$; for each $v \in V, \bar{x}_{i j}^{t, v}$ and $\underline{x}_{i j}^{t, v}$ are the optimal solution of two minimum cost flow problems $\overline{S P}_{v}^{t}$ and $\underline{S P}_{v}^{t}$, modeling the shortest upstream and downstream gateway paths $\bar{p}_{v}^{g t w(t, v)}$ and $\underline{p}_{v}^{g t w(t, v)}$.

Now we extend the static formulation to a dynamic setting, where demand varies with time. Consider $t$ as one of the $n_{T}$ time periods in which the time horizon $T$ is divided, i.e., $T=\left\{1, \cdots, n_{T}\right\}$. Gateways location and allocations decisions vary at each period, according to $\varphi_{v}^{t}$. In order to capture the periodic schedule of shipments, we suppose that the authority receives the full list of the trips due at period $t$ at the end of period $t-1$. In the most flexible case, all decisions are taken independently at the beginning of each $t$. This corresponds to solving a separate $P^{G T W}(t)$ problem at each $t$. This option yields the highest infrastructure cost, as it requires to relocate all the gateways that have not been confirmed from $t-1$ to $t$, but also provides the highest risk mitigation achievable in a GLP based system. We call this scenario the $k$-flexy. Take as the Infrastructure Flexibility Index $(I F I)$ the maximum number of gateways that can be relocated, denoted by $c$. By varying $c \in[0, k]$ and given $\hat{y}_{h}^{t-1}$ the solution of the previous period $t-1$, we can formulate the problem faced at time $t$ in the $c$-flexy scenario, that we call $P^{G T W}(t, c)$, as the generic formulation for period $t$ given above plus constraint (8) 


$$
\sum_{h \in \hat{H}^{t-1}} y_{h}^{t} \geq k-c
$$

where $\hat{H}^{t-1}$ is the index set of the gateways selected at $t-1$, i.e., $h \in \hat{H}^{t-1}$ iff $\hat{y}_{h}^{t-1}=1$. Problem $P^{G T W}(t, c)$ can be reformulated as a single level MILP problem, according to the same arguments discussed in [4], and therefore solved by state of the art MILP solvers.

\section{$3 \quad$ Experimental results}

The aim of the field trial and to evaluate the impact of the IFI on the reduction of risk mitigation due to variable demand.

First we describe the test instance generation and how results have been analyzed, then conclusions are drawn and future work is sketched. The testbeds have been derived from a widely used instance, namely the Ravenna network $([2,4])$. It provides an indirected graph with $|N|=105$ nodes and $|A|=134$ arcs, being an abstraction of the road network of the city of Ravenna (Italy), a cost and a risk function defined on the arcs. In particular, the risk function is the so called aggregate function whose values are based on the frequency of hazmat release in case of accident times a population figure related to places of assembly within 500 meters of the arc. This network has been transformed into a directed network in standard way. The static travel demand is made of $|V|=35$ vehicles with their origin-destination pairs and shipment requests. On this instance we have already tested the risk mitigation potential of the GLP based method and we have experimentally identified $k^{s t a b}$, the minimum number of open gateways necessary to capture the most of it ([5]). In particular, for the aggregate risk function such value is 5 , setting the range for the $I F I$ as $c \in[0,5]$. Moreover, in this campaign we consider any node in $N$ as a potential site for a gateway so that no decision on the candidate sites can interfere with the experiments. These data form the starting point for the generation of the sets of dynamic instances. We assume that all shipments concern the same material and that the 35 demand values provide a random sample extracted from the same population. Looking at the descriptive statistics of the sample reported in Table 1, it can be argued that demand is positively skewed and characterized by a large standard deviation. To identify a suitable distribution we applied the Anderson Darling goodness-of-fit test to several non-negative and positively skewed distributions (namely, Exponential, Lognormal, Weibull, Logistic, Loglogistic, and Gamma). The best fitting one is a $\operatorname{LogNormal}$ distribution with parameters $\mu=6.719$ and $\sigma=1.957$, 
Table 1

Descriptive statistics of the sample provided by the static demand

\begin{tabular}{|l|r|r|r|r|r|r|r|}
\hline Size & Mean & StDev & Median & Min & Max & Skewness & Kurtosis \\
\hline 35 & 4216.89 & 9127.45 & 995 & 25 & 50186 & 4.08052 & 19.509 \\
\hline
\end{tabular}

for which we obtained a test statistics $A D=0.363$ and a $p-$ value $=0.421$. Sampling from this distribution we build a set $S$ of $n_{D I}=30$ dynamic instances, assuming one year as the time horizon $T$ and one month as the time period $\left(n_{T}=12\right)$, so that each dynamic instance $s \in S$ is a matrix of $n \times n_{T}$ integers representing the demand values $\varphi_{v}^{t}(s)$ for each vehicle $v \in V$ and time period $t \in T$. Data are available from the authors upon request. For each instance $s$ and for each value $c$ of the $I F I$, we solve $n_{T}$ problems $P^{G T W}(t, c)$ with demand $\varphi^{t}(s)=\left\{\varphi_{v}^{t}(s) \forall v \in V\right\}$. The MIP model is solved by CPLEX 12.1 on a AMD Athlon(tm) 64 X2 Dual Core Processor 4200+; average running time is 0.04 seconds, excluding the preprocessing phase whose duration is negligible. For each problem, the risk value is normalized with respect to the case $c=5$, which provides an upper bound to the minimum risk achievable by the GLP method, thus yielding a measure of risk deterioration. Note that the phis are independent i.e. they were generated sampling from the same distribution but no correlation exists between the samples. On the contrary, for each $s$, when $c=0$ the gateway set $N^{t}$ is fixed and depends only on $\varphi^{1}(s)$ while, when $c \geq 1, N^{t}$ at $t>1$ is influenced by $\varphi^{t}(s)$ as well as by $N^{t-1}$, i.e., recursively, by all demands at the previous time periods.

First, we analyze robustness with respect to variable demand. For each $t$ and for a given $c$, we use the $n_{D I}$ normalized risk values to compute a $95 \%$ confidence interval for the mean, depicted in the interval plot in Figure 1. For $c=0$, despite the fact that the gateway set does not vary, the upper bound of the confidence interval is below $12 \%$. However, relocating at most one gateway at each time period (i.e. $c=1$ ), on the one hand reduces the mean value of the normalized risk, on the other hand it considerably reduces its standard deviation. As a whole, when moving from $\mathrm{c}=0$ to $\mathrm{c}=1$ the confidence intervals for the mean shrink and shift toward 1, while updating at most two gateways at each $t$ is sufficient to flatten risk deterioration to zero. Therefore, we can conclude that the GLP method is quite robust with respect to demand variations as far as the origin and destination of the shipments stay the same. This is a reasonable assumption since the location of any hazmat place of production or utilization is a long period decision. Moreover, the case $c=0$ can also be seen as the case of unpredictable demand, which is quite unlikely 
since HazMat shipments data must usually be reported to the authority in advance. From a mathematical point of view, such results come at no surprise, as we observe that the risk of a gateway path is not influenced by demand. Rather, demand acts as a weight factor of such risk, favoring the selection of those gateways that, if chosen, most reduce the risk of the largest shipments. In other words, if $k$ were equal to $n$, that is, if each vehicle could select its own best gateway, demand would not affect solution quality.

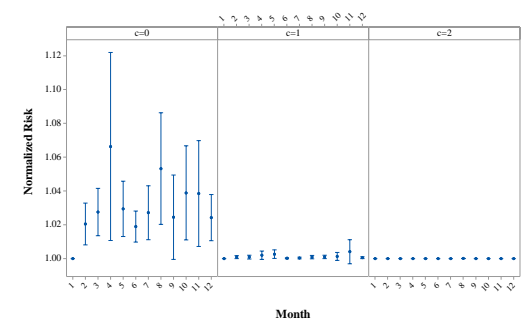

Fig. 1. Interval plot of the $95 \%$ confidence interval for the mean

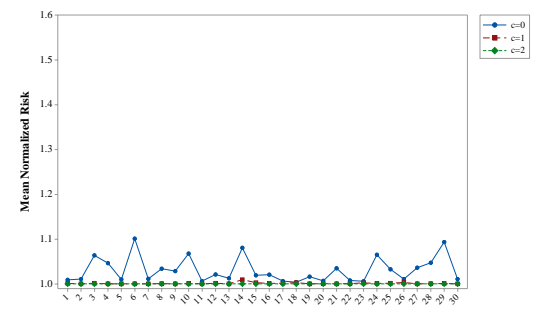

Fig. 2. Mean normalized risk deterioration over the 12 time periods for each instance $s=1 . .30$

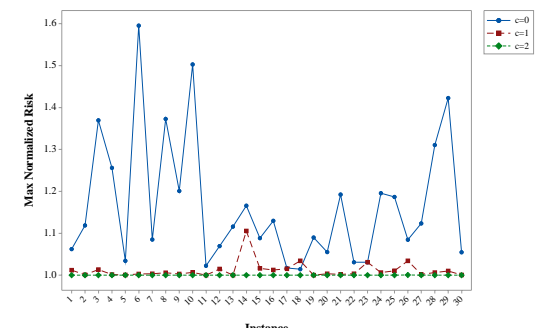

Fig. 3. Maximum normalized risk deterioration over the 12 time periods for each instance $s=1 . .30$ 
Another kind of analysis can be done by plotting for each $s$ the mean and the maximum risk deterioration over the entire horizon $T$, again comparing the rigid case $c=0$ with the cases $c=1$ and $c=2$. If flexibility is not allowed, there may be substantial risk deterioration at individual instances, which justifies the need for sensitivity analysis. On the one hand, it is well expected that a higher flexibility allows for a lower loss of risk mitigation; on the other hand, the cost of full flexibility would be unbearable for large instances. Therefore, a trade off must be sought and this requires the knowledge of how sensitive risk mitigation is with respect to $c$. Results suggest that $c=2$ is enough for this class of instances. On going work is assessing this finding on other networks and risk functions.

This study spurs further research along three lines: (i) the way set $N^{t}$ varies with $t$ could suggest gateways selection policies to be adopted when no infrastructure flexibility is allowed but demand may vary; (ii) robust optimization could be investigated if interested in hedging against poor system performance for some demand realization; (iii) assuming the a priori deterministic knowledge of the demand for each time period, a one-shot formulation encompassing the whole planning horizon could be tackled by decomposition approaches.

\section{References}

[1] Batta,R., and C. Kwon, "Handbook of OR/MS Models in Hazardous Materials Transportation", Int. Series in O.R. \& Management Science, (2013) Springer.

[2] Erkut E, and F. Gzara, Solving the hazmat transport network design problem Computers and Operations Research 35 (2008), 2234-2247.

[3] Kara, B.Y., and V. Verter, Designing a Road Network for Hazardous Materials Transportation, Transp. Science 38(2), (2004) 188-196.

[4] Bruglieri, M., P. Cappanera, A. Colorni, and M. Nonato, Modeling the gateway location problem for multicommodity flow rerouting, in "INOC 2011", Lecture Notes in Computer Science 6701, (2011) 262-276, Springer.

[5] Bruglieri M., P. Cappanera, and M. Nonato, The Gateway Location Problem: Assessing the impact of candidate site selection policies, Discrete Applied Mathematics 165 (2014), 96-111.

[6] Cappanera P., and M. Nonato, The Gateway Location Problem: A Cost Oriented Analysis of a New Risk Mitigation Strategy in Hazmat Transportation, Procedia - Social and Behavioral Sciences 111 (2014), 918-926. 